\title{
DESIGUALDAD Y PERSONAS MAYORES DE 60 AÑOS EN LA CIUDAD DE ARMENIA, QUINDÍO
}

\section{INEQUALITY AND PEOPLE OLDER THAN 60 YEARS IN THE CITY OF ARMENIA, QUINDÍO}

\author{
Néstor Raúl Giraldo Navia ; Álvaro Alfonso Fernández Gallego;
}

John Jaime De la Rosa Bobadilla

Fecha de recibido: Febrero 3 de 2010

Fecha de aceptado: Junio 9 de 2010

Correspondencia: Programa de Gerontología, Universidad del Quindío Av. Bolivar calle 12 norte Armenia Quindío. Correo electrónico: luar666@gmail.com.

\section{RESUMEN}

Por las características exploratorias y focalizadas de la investigación "Condiciones de vida de los adultos mayores de la ciudad de Armenia", la desigualdad será medida a partir del ingreso, los aportes económicos de las personas mayores al hogar y los cambios en los consumos, como variables de aproximación a ella, incluso con las limitaciones que impone. En correspondencia con trabajos de la Comisión Económica para América Latina (CEPAL), el aporte de las personas mayores de 60 años para el sostenimiento y reproducción económica de los hogares es considerable, desde un casi $50 \%$ en adelante y de modo absoluto para los hogares situados en las franjas de Pobreza y Pobreza Extrema. El aporte, en la medida en que es mayor el ingreso del hogar, disminuye el aporte alto de los cabezas de hogar mayores, pero tiende a aumentar el aporte medio de manera importante. Esto configura unos arreglos familiares de corresponsabilidades económicas directas entre los miembros mayores y los menores de 60 años. En cuanto a los consumos, leídos a través de los ingresos, resulta mucho más sensible para entender el comportamiento económico de los hogares, desde el que es dable observar, independiente de las tres categorías de No Pobre, Pobre y Pobreza Extrema, ya analizadas (Giraldo, Fernández y De la Rosa, 2008), y que han arrojado resultados consistentes, pero en este caso específico no tan precisos como ahora.

Palabras claves: desigualdad, pobreza, aportes económicos, ingresos, consumos y personas mayores.

\begin{abstract}
By the exploratory and focalized characteristics of the investigation "Living conditions of the old age people of the city of Armenia", the inequality was measured from income and economic support of these old age people to their homes and the changes in consumption, as variables of approximation to inequality with the limitations that it imposes. According with works of the Economic Commission for Latin America (CEPAL), the contribution of people older than 60 years to the economic sustainability of their homes is considerable, from almost 50\% hereinafter and for homes located in the borders of poverty and extreme poverty. The economic contribution, having in mind that the home's income are greater, decreases the high economic contribution of the household head, but it tends to increase the average intakes in an important manner. This sets up some familiar arrangements of direct economic co-responsibilities between senior members and members younger than 60 years old. When it comes to consumptions, in terms of income, it results a lot more sensitive to understand the economic behavior of homes, from which is observable three independent categories of No poverty, Poverty and Extreme Poverty, already analyzed (Giraldo, Fernández and De la Rosa, 2008), and that have showed consistent results, but in this specific case not as precise as now.

Key words: inequality, poverty, economic contribution, income, consumption and older people.
\end{abstract}

\section{INTRODUCCIÓN}

El enfoque de desigualdad aplicado a las personas mayores presenta cierta tendencia a considerar "la vejez como un tiempo de 'estanco', estático, que no se condice con la concepción de vejez como un proceso durante el cual "el individuo continúa dialogando con las estructuras sociales y económicas" (Pérez, 1997).

La ampliación del enfoque de desigualdad (Aranibar, 2001, 31-38), en términos generales y para todos los grupos etáreos, se asocia a la persistencia de la pobreza y se vincula de manera específica al ajuste y a las reformas económicas de los años ochenta, así como a la posterior consolidación del patrón de desarrollo vigente (Pizarro, 1999).

Las estimaciones más recientes permiten afirmar que en las zonas urbanas la incidencia de la pobreza afecta tanto a hombres como mujeres, sin un patrón diferencial según género. Por otra parte, en las áreas rurales es evidente que la pobreza afecta de forma relativa más a las mujeres que a los hombres mayores (Del Popolo, 2000), y en general, a los hogares rurales

Grupo de Investigación en Desarrollo, Universidad del Quindío.

${ }^{2}$ Realizada con recursos de la Vicerrectoría de investigaciones de la Universidad del Quindío, en el año 2005. 
sobre los urbanos en la región del Eje Cafetero (Informe Regional de Desarrollo Humana - IRDH: "Un Pacto por la Región, PNUD, 2004), donde se inscribe este trabajo.

Las perspectivas de la desigualdad, la dependencia y la vulnerabilidad, hacen parte de la rica discusión, reinaugurada hacia la década del 70 en adelante, y formalizada hacia los 90 por el Programa de las Naciones Unidas para el Desarrollo (PNUD), sobre la valoración y medición de la pobreza, y la calidad de vida, e introducida por diversos economistas, profesionales de las ciencias sociales y de la salud, e intelectuales influyentes, entre los que se destacan los aportes de Jhon Rawls con A Theory of Justice (1971), y la ricas y novedosas perspectivas de los trabajos de Amartya Sen, como el expresado en una conferencia en 1979 “¿lgualdad de Qué?", que han permitido institucionalizar un debate que estuvo dominado por el planteamiento unilateral de la escuela clásica y neoclásica, con la teoría utilitarista y sus vertientes y versiones, y estigmatizado, ocultado o desacreditado, desde el bipolarismo, cuando se abordaba desde el marxismo ortodoxo predicado por los países socialistas, y en particular por la cortina de hierro occidental.

Por ello se dice acerca de este debate como reinaugurado, público y publicitado, sobre el silencio de los aportes de las teorías críticas y marxistas, retomado ahora por intelectuales, que encareciendo el desarrollo histórico occidental, valorando lo que consideran sus logros, y afirmados en ellos, cuestionan las bondades automáticas del mercado, del crecimiento económico y del desarrollo económico. Es así como la desigualdad, la inequidad y la exclusión se convierten en conceptos que matizan y profundizan las tradicionales concepciones de pobreza, y en particular, la hegemonía en su medición, establecida y desarrollada por el Banco Mundial, centrada en los niveles de ingreso.

Sin desestimar estas medidas, lo que plantean la mayoría de índices (Seguridad Humana, trabajo decente, Índice de Desarrollo Humano, Capacidad...), es la ampliación de su lectura, contrastando con otras dimensiones de bienestar distintas a los recursos económicos, o la de las condiciones materiales.

La desigualdad entonces es entendida, más allá de un estándar básico único, como una medida relativa, que en su acepción más simple, se puede interpretar como la dispersión de una distribución, sea del ingreso, como del consumo o de algún otro indicador de bienestar o atributo de una población.

En ausencia de trabajos actualizados distintos al IRDH "Un Pacto Por la Región" (PNUD, 2004), sobre los ingresos, se expresará esta desigualdad al interior del marco poblacional de este estudio (Hogares con presencia de adultos mayores en la ciudad de Armenia).

Por las características exploratorias y focalizadas del estudio, para este aparte sobre la desigualdad, serán el ingreso, los aportes de las personas mayores al hogar, y los cambios en el consumo las variables de aproximación a ella, con las limitaciones que imponen.

Pues como ya se menciono, la calidad de vida y el bienestar son construcciones multidimensionales que involucran dimensiones, en términos de Sen, de capacidades y libertades para allegar logros, bajo la consideración de lo que para la gente es, en perspectiva, una buena vida, distinguiendo aquellas desigualdades que no son inequidades, valga decir, diferencias (color de ojos, étnia, gustos de lectura...) de aquellas otras que si lo son, es decir, que son inaceptables (apropiación y distribución inequitativa de la riqueza socialmente producida; exclusión política, laboral, en razón del sexo, la etnia, o el credo), y que de un modo $u$ otro son "diferencias que se consideran injustas y evitables" (López A. Oliva, en línea 19/07/2006, 14:30).

Habría que agregar sin embargo, dos factores interdependientes: El primero, es el factor estructural, la producción y la reproducción de una sociedad están marcadas y estructuradas desde su inicio para cada generación, por unas condiciones de distribución y poder que asignan, limitan o niegan el acceso a los recursos para una vida digna o éticamente valorada; y segundo, como una consecuencia de esta desigualdad o inequidad estructurante, con frecuencia, "debido a la forma en que los deseos pueden ser limitados de por vida por privaciones y falta de educación, de modo que las expectativas se ajustan a condiciones de vida subnormales (...)" (Jayawardena L. 1990).

\section{METODOLOGÍA}

Primero: la investigación se realizó por medio de una muestra de 384 hogares (un total de 1439 personas), para un error de muestreo del $5 \%$ y una confianza del $95 \%$. Los datos fueron obtenidos a través de la aplicación de una encuesta social que contiene 104 preguntas y está organizada por secciones y subsecciones (Giraldo et al, 2008, 114-115). Los datos se almacenaron en una base ACCES y se procesaron en el software STATGRAPHICS versión 5.0.

Segundo: la estimación de la pobreza por el Índice de Necesidades Básicas Insatisfechas (NBI), como por Condiciones de Vida (ICV), en primera instancia ha permitido, como ya se ha planteado, generar una primera desagregación de los hogares con personas mayores de 60 y más años en la ciudad de Armenia, sobre tres categorías: Hogares No Pobres, Hogares Pobres y Hogares en pobreza extrema ${ }^{3}$.

Tercero: esta aproximación proyecta una primera perspectiva de los hogares con mayores y su distribución sobre una escala de desigualdad y bienestar, entendido éste en términos restringidos, como acceso a recursos.

${ }^{3}$ Ver Giraldo N, N.R. et al (2008). Dependencia económica y social de adultos mayores de 60 años en la ciudad de Armenia, Quindío. Revista de Investigaciones No.18, Universidad del Quindío, Armenia.

Rev. Invest. Univ. Quindío (20): 55 - 65. Armenia - Colombia 
¿Por qué los aportes de las personas mayores? Con regularidad, al hablarse de pobreza y desigualdad, al tomar en consideración los hogares, entre otras variables -educación, número de integrantes, ingresos- se considera que los hogares presididos por personas mayores, o con estas personas, son más vulnerables y más intensamente pobres, en tanto la dependencia económica se manifiesta con la vejez.

En sociedades en plena transición demográfica, el aumento de la esperanza de vida no solo presiona el sistema de seguridad social de una nación y obliga nuevas redistribuciones; sino, que impacta en primer lugar sobre los hogares con esta población. Sobre estas tendencias (Fresneda, O. 1991), consideradas como nociones que se estereotipan, hay también un debate en contrario que plantea que según sea la organización social (incluida por supuesto la política y la política laboral), sin desmedro de los ingresos y el trabajo de la PET (población en edad de trabajar), se logran óptimos para estos grupos de edad mayores asegurando ingresos, y no solo erogaciones.

Sin embargo, lo contrario no solo es posible, sino que, de manera muy importante, es sobresaliente; hallazgo ya expresado por CEPAL-ECLAC (2000) en varias oportunidades. Además, por efecto de esta misma transición demográfica, la conjugación y la interacción de varias generaciones se hacen más probables e intensas, tributando en un mismo hogar, mucho más que antes.

Como lo plantea la consultora Fabiana del Popolo:

"Un importante efecto del envejecimiento es su impacto en la composición de los hogares y los arreglos familiares. En el pasado a causa de la elevada fecundidad y las menores expectativas de vida era poco común un hogar compuesto por más de dos generaciones. Pero los cambios demográficos de las últimas décadas hicieron más probable que una familia tenga tres o cuatro generaciones; los arreglos residenciales y la relación multigeneracional son más complejos" (2000).

Tercero: Fabiana del Popolo (2000), expone la división de los hogares multigeneracionales en tres grupos, según el monto del aporte de la persona mayor considerada la totalidad del ingreso del hogar, en Alto Aporte, cuando los ingresos aportados son más de la mitad del ingreso del hogar: Aporte Medio, cuando representan de 25 a 50\%; y Aporte Bajo, cuando es inferior al $25 \%$ del total del ingreso del hogar.

\section{RESULTADOS Y DISCUSIÓN}

Diferente a los hogares de constitución reciente, el ciclo de vida de los hogares de Armenia según la muestra, en una alta proporción son presididos por personas mayores, lo que hace que la pirámide de edad sea diferenciada, a pesar de la multigeneracionalidad, y también mejor, precisamente por ella, de las pirámide de base más ancha y cúspide más estrecha.

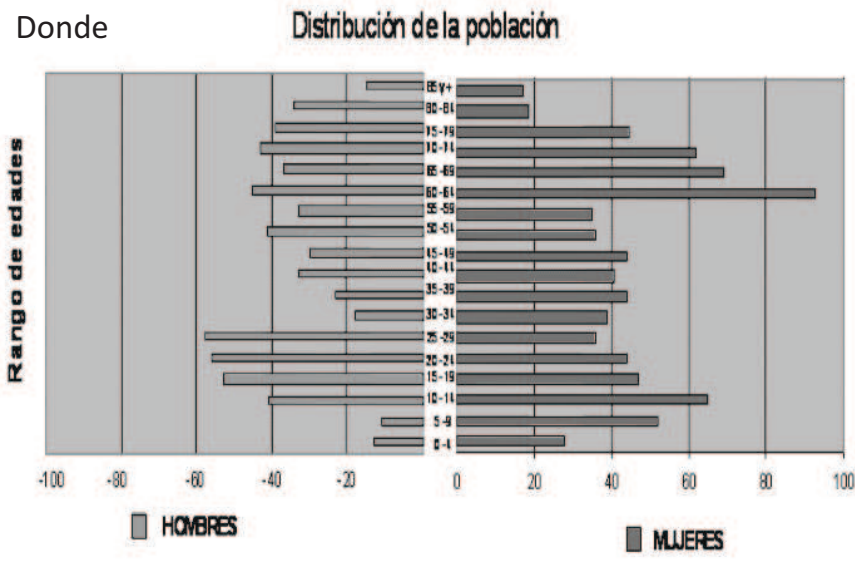

Figura 1. Distribución de la población (muestra).

- Es visible, la mayor proporción de mujeres de todas las edades sobre las de hombres

- Los siete grupos superiores de edad, es decir de 60 años en adelante concentra las mayores proporciones en conjunto, con una proporción mayor de mujeres (sobreviven a su compañero).

- Los grupos de edad desde los 10 a los 29 años representan la segunda proporción en importancia, en estos hogares.

- Los grupos de 0 a 9 años corresponden a los de menor proporción (Giraldo, et al, 2008, 116).

El cuadro siguiente presenta el parentesco de los miembros de los hogares y sin corresponder necesariamente a menores de edad, uno de los cuatro grupos casi equivalentes en proporción que le siguen en importancia al grupo hijos o hijastros, lo constituyen las nietas y los nietos.

Tabla 1. Parentesco de los miembros del hogar.

\begin{tabular}{lcc}
\hline Parentesco & Cantidad & \% \\
\hline Jefes de hogar & 384 & 27 \\
Compañeros(as) & 217 & 15 \\
Hijos o hija stros & 371 & 26 \\
Yernos/nueras & 42 & 3 \\
Nieto(a) & 182 & 13 \\
Abuelo(a) & 34 & 2 \\
Otro Pariente del & 201 & 14 \\
jefe & 8 & 1 \\
Otro no pariente & $\mathbf{1 4 3 9}$ & $\mathbf{1 0 0}$ \\
Total & & \\
\hline
\end{tabular}

Rev. Invest. Univ. Quindío (20): 55 - 65. Armenia - Colombia 
Para contextualizar de manera más precisa y clara, el gráfico anterior establece la proporción en que los adultos mayores presiden sus hogares como cabezas de hogar y la consecuente importancia de la fracción que representa su aporte económico al conjunto del hogar.

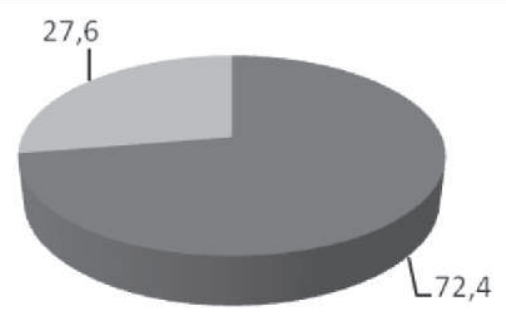

- Hogares con Jefes mayores Hogares sin jefes mayores

Figura 2. Hogares con jefes mayores, (\%).

Desagregando por jefatura masculina y jefatura femenina, se tiene de los adultos mayores que presiden como cabezas o jefes de hogar, un $63 \%$ de jefatura masculina de persona mayor, y un $37 \%$ de jefatura femenina de persona mayor.

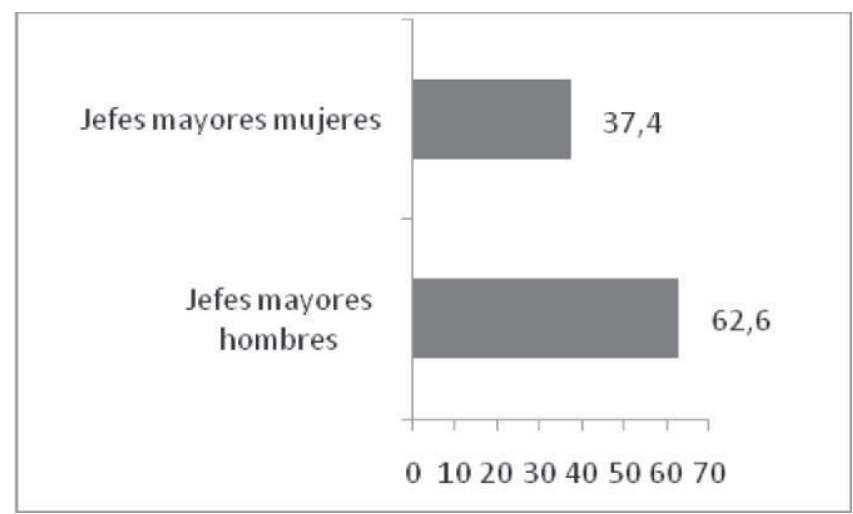

Figura 3. Aportes económicos de los jefes mayores por sexo (\%).

Incluida la totalidad de los hogares, los aportes del viejo o adulto mayor, en su calidad de jefe, es la siguiente:

Tabla 2. Aportes de los jefes mayores.

\begin{tabular}{lc}
\hline APORTE JEFES MAYORES & $\begin{array}{c}\text { TOTAL } \\
\text { HOGARES }\end{array}$ \\
\hline Alto & 30,99 \\
Medio & 14,84 \\
Bajo & 1,3 \\
Sin aporte & 52,86 \\
TOTAL & 100 \\
\hline
\end{tabular}

Rev. Invest. Univ. Quindío (20): 55 - 65. Armenia - Colombia
Lo que representa que casi el $50 \%$ de los hogares reciban aportes, que el $31 \%$ de todos los hogares basen su reproducción económica de los adultos mayores; y que una sexta parte (15\%), dependa en un aproximadamente $50 \%$ de los ingresos totales de sus hogares, del aporte del adulto mayor.

Tabla 3. Aportes al hogar de jefes mayores según categoría de pobreza.

\begin{tabular}{lccc}
\hline $\begin{array}{l}\text { Tipo de } \\
\text { aporte }\end{array}$ & $\begin{array}{c}\text { Hogares No } \\
\text { Pobres \% }\end{array}$ & $\begin{array}{c}\text { Hogares } \\
\text { pobres \% }\end{array}$ & $\begin{array}{c}\text { Hogares en } \\
\text { extrema } \\
\text { pobreza \% }\end{array}$ \\
\hline Alto & 30,86 & 29,09 & 60 \\
Medio & 14,81 & 14,55 & 20 \\
Bajo & 1,54 & 0 & 0 \\
Sin aporte & 52,78 & 56,36 & 20 \\
Total & 100 & 100 & 100 \\
Total & & & \\
hogares & 324 & 55 & 5 \\
\hline
\end{tabular}

Desagregado por los tres grupos, es claro que los hogares presididos por personas mayores dependen para su subsistencia, en alguna proporción, desde baja hasta alta, en los Hogares No Pobres, en un 47,22\%; en los Hogares Pobres, en un $44 \%$; y en un $80 \%$ en los Hogares que se encuentran en pobreza extrema (aclarando por supuesto que la muestra es muy pequeña en este último caso).

También queda claro con la figura 3, que las proporciones de mayor dependencia del hogar del aporte del adulto mayor, por representar su aporte la totalidad o casi la totalidad del ingreso del hogar, se mantiene muy similar entre No Pobres y Pobres, pero asciende hasta duplicarse en el grupo en Pobreza Extrema, lo que manifiesta:

-Una vulnerabilidad obvia y creciente a medida que se desciende a los Hogares en Miseria. De manera similar se comporta la dinámica con los aportes medios al hogar por parte del adulto mayor.

- Los cabeza de hogar de los mayores de edad representan una proporción considerable en su propio grupo (No Pobre, pobre, y en pobreza extrema), algo más baja en el grupo de Pobres que el de No Pobres, pero si absoluta en los de Pobreza Extrema.

- No se encuentran diferencias apreciables en los grupos de mayor proporción (No Pobres y Pobres) en los montos de sus aportes.

- La manera en que el N.B.I mide en líneas gruesas, en una región y ciudad urbana y materialmente reconstruidas post-evento sísmico del año de 1999, y con una trayectoria de servicios básicos casi universal producto de una larga 
estabilidad cafetera hasta la caída del pacto, y bajo el amparo de políticas ambientales y sociales de la Federación de Cafeteros, auténtico para estado en el auge y estabilidad cafeteras, hacen que algunas variables del I.C.V. sean casi irrelevantes, 0 insensibles en general, salvo en sus extremos inferiores, para calificar pobreza.

Estas proporciones de aportes, de manera más pormenorizada y sensible se podrán apreciar cuando la lectura se haga desde los ingresos por salarios de los hogares y ya no por las tres categorías anteriores.

\section{Hogares No Pobres según aportes}

La figura 4 de manera simple muestra, que allí donde se da aporte de los cabezas de hogar mayores de edad, se da una progresión: en los hogares con jefes de aporte alto, el de hombres casi duplica el de mujeres; en los de aporte medio, casi triplica el hombre; y en los de bajo aporte, el peso masculino es cuatro a uno por sobre las cabezas de hogar femeninas, que ya sobre el conjunto del grupo total no es posible apreciar. Los grupos de bajo aporte y sin aporte significan dependencia económica de las personas mayores de otras personas en el hogar, equivalentes al 30,24\% de mayores jefes dependientes.

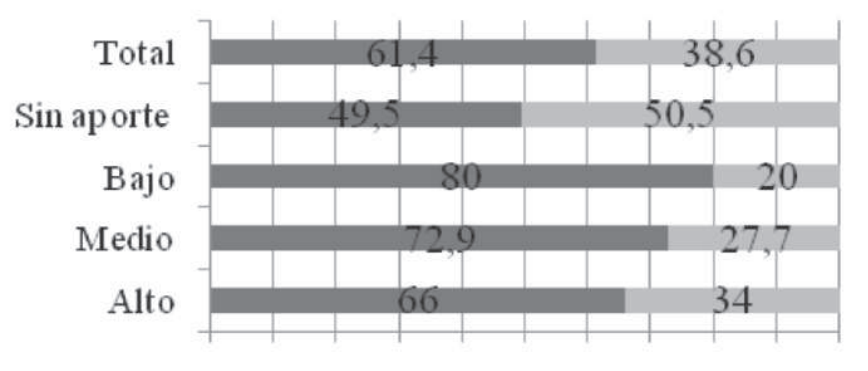

$\begin{array}{lllllllllll}0 & 10 & 20 & 30 & 40 & 50 & 60 & 70 & 80 & 90 & 100\end{array}$

- Hombres Mujeres

Figura 4. Aportes por sexo jefes de hogar No Pobres (\%)

Tabla 4. Aportes por sexo y total grupo No Pobres (\%)

\begin{tabular}{ccccccc}
\hline Sexo & Alto & Medio & Bajo & Subtotal & $\begin{array}{c}\text { Sin } \\
\text { aporte }\end{array}$ & $\begin{array}{c}\text { Sin } \\
\text { información }\end{array}$ \\
\hline Hombres & 20,37 & 10,8 & 1,23 & 32,41 & 14,2 & \\
Mujeres & 10,49 & 4,01 & 0,31 & 14,81 & 14,51 & \\
Total & $\mathbf{3 0 , 8 6}$ & $\mathbf{1 4 , 8 1}$ & $\mathbf{1 , 5 4}$ & $\mathbf{4 7 , 2 2}$ & $\mathbf{2 8 , 7}$ & $\mathbf{2 4 , 0 8}$ \\
\hline
\end{tabular}

Tabla 5. Aportes jefes mayores grupo No Pobres (\%).

\begin{tabular}{lcccc}
\hline Tipo de aporte & Hombres & Mujeres & $\begin{array}{c}\text { Total } \\
\text { Hogares } \\
\text { jefes } \\
\text { mayores }\end{array}$ & $\begin{array}{c}\text { Total } \\
\text { Hogares } \\
\text { Grupo }\end{array}$ \\
\hline Alto & 26,83 & 13,82 & 40,65 & 30,86 \\
Medio & 14,23 & 5,28 & 19,51 & 14,81 \\
Bajo & 1,63 & 0,41 & 2,03 & 1,54 \\
$\begin{array}{l}\text { Subtotal sexo } \\
\text { con aportes }\end{array}$ & $\mathbf{4 2 , 6 9}$ & $\mathbf{1 9 , 5 1}$ & $\mathbf{6 2 , 2}$ & $\mathbf{4 7 , 2 2}$ \\
$\begin{array}{l}\text { Sin aporte total } \\
\text { jefes }\end{array}$ & & & & \\
$\begin{array}{l}\text { Sin aporte total } \\
\text { grupo }\end{array}$ & & & $\mathbf{3 7 , 8}$ & \\
$\begin{array}{l}\text { Sin } \\
\text { información }\end{array}$ & & & $\mathbf{2 8 , 7}$ \\
\begin{tabular}{l} 
Total \\
\hline
\end{tabular} & & & $\mathbf{1 0 0}$ & $\mathbf{1 0 0}$ \\
\hline
\end{tabular}

El 76\% de cabezas de hogar del grupo de No Pobres, está constituido por personas mayores; de este grupo de cabezas aportan al hogar con aporte alto, medio o bajo el $62,2 \%$ del subgrupo de No Pobres con jefes mayores, que a su vez representan el $47,2 \%$ de cabezas de hogar de todo el grupo de No Pobres.

Cabe recordar que la tabla según nivel de aportes para los tres grupos está ponderado de acuerdo a la totalidad de hogares de la muestra, mientras las cifras mencionadas en el párrafo anterior corresponden sus porcentajes al número de hogares del grupo, en este caso de No pobres, y ya no sobre la totalidad de los hogares o sumatoria de los tres grupos, revelando ahora que el peso para el grupo específico es mayor ahora al pasar de 39, 845 (40\%), a 47,2\%.

Una vez establecidas estas proporciones, la tabla permite establecer el peso por sexo total y por niveles, como se puede observar, donde se mantiene como aportante mayor al hombre en todos los casos, salvo en el caso de no aportantes económicos donde son en general, equivalentes.

De igual manera se observa que a medida que desciende el aporte de alto hacia bajo, aumenta el número de aportantes masculinos, ocurriendo lo contrario para las mujeres; es decir, que aportan más mujeres en el nivel alto, disminuyendo hasta llegar a aporte bajo.

Sintetizando, del total de hogares no pobres, con jefes mayores y no mayores, las personas mayores contribuyen 
con algún nivel de aporte hasta el 47, 22\%, que asciende a $62,20 \%$ al tomar del grupo general de no pobres el subgrupo de jefaturas de mayores, donde el $37,8 \%$ de estas jefaturas no hacen aporte económico a sus hogares; es decir, que visto desde este subgrupo de jefaturas mayores, la dependencia es mucho más intensa, sin mencionar aquellas personas mayores no jefes.

Discriminando por sexo, los hogares de jefaturas de personas mayores está constituida en un $61,38 \%$ por hombres, y en un $38,62 \%$ de mujeres: la dependencia en este caso se presenta entre los hombres mayores para el $33,11 \%$, en tanto para las mujeres es mucho más extensa, al alcanzar al 50,53\% de las que son jefes de hogar. Se ha tomado dependencia económica sumando bajo aporte y sin aporte, para cada uno de los sexos

\section{Hogares pobres según aportes}

Para este grupo de Hogares Pobres, aumenta la importancia del aporte de los jefes mayores, en la medida en que sus aportes representan, en términos absolutos, al interior del subgrupo de jefaturas de adultos mayores el $85,7 \%$ frente a $62,2 \%$ en los no pobres; mientras el aporte masculino asciende de $43 \%$ en los no pobres, a $68 \%$ en los pobres, y en las mujeres de $19,5 \%$ de no pobres cae a $18 \%$, pero esta aparente disminución lo es en tanto que la composición de las jefaturas por sexo varía en los pobres, al aumentar la proporción de jefatura masculina en los hogares presididos por mayores de $61,4 \%$ en no pobres a $71,4 \%$, y en las mujeres se pasa de $38,6 \%$ no pobres a $28,6 \%$ en pobres; que en términos relativos significa pasar de un aporte de $49,5 \%$ en no pobres a $62,5 \%$ en los pobres por parte de las cabezas de hogar femeninas. Fenómeno social que se corresponde con los hallazgos de la CEPAL en Panorama Social 1999 - 2000, y Paula Aranibar (2001).

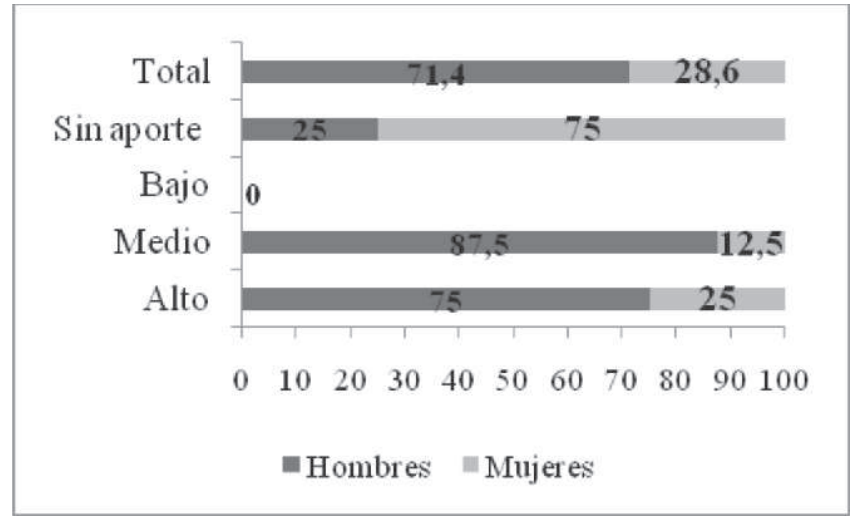

Figura 5. Aportes por sexo Jefes de Hogar Pobres (\%)

De otra parte, las jefaturas femeninas alcanzan a un $37,5 \%$ que no tienen ingresos propios para hacer algún aporte económico, mientras para los hombres esta proporción equivale a un $5 \%$.

En los casos que se vienen analizando, es manifiesta la mayor desigualdad y consecuente vulnerabilidad de los hogares presididos por mujeres, aunque la dependencia económica varía de manera muy importante en estos grupos, al caer para los dos sexos de hogares no pobres para hombres de $33,1 \%$ a $5 \%$ en pobres; y de $50,5 \%$ de dependencia de las mujeres en no pobres, a $37,5 \%$ en pobres.

Visto desde la otra parte -la dependencia económica inversa-, la de los hogares respecto del aporte de sus jefaturas mayores, es menor en los hogares no pobres que en los hogares pobres.

En términos generales, la afectación por inestabilidad laboral y de ingresos, es mucho mayor en los hogares de pobres, con su bajo nivel escolar, sus fuentes informales de trabajo, y sus menores ingresos para costear las demandas de sus hogares. Menos hogares de los hogares no pobres dependen del aporte alto y medio de sus cabezas $(60,2 \%)$, que en los pobres (85,7\%), o como afirma Paula Aranibar:

"Si se considera que las condiciones de vida (en su sentido amplio) de las personas mayores pueden verse amenazadas cuando algún miembro de la familia experimenta cambios en su situación laboral (usualmente, merma en el ingreso), se supone unidireccionalidad de los flujos de apoyo e intercambio, desde los adultos jóvenes hacia las personas mayores, sin considerar los aportes que hacen los ancianos al grupo familiar; sin embargo, el análisis de los datos muestra una realidad diferente" (2000).

Aun cuando parezca extraño, los adultos mayores sostienen buena parte de esos hogares; más aún, la ausencia de personas de edad aumentaría la incidencia de la pobreza a niveles elevadísimos, en particular en aquellos hogares donde el aporte del adulto mayor supera la mitad del ingreso total (Panorama Social, 1999-2000, 46).

\section{Hogares en pobreza extrema según aportes}

En el caso de los Hogares en Pobreza extrema, dependen estos del aporte de ambos sexos, aunque con la señalada tendencia masculina de aportante principal; sin embargo, para este grupo no se encontraron adultos mayores que no hicieran aportes económicos, y esta es una diferencia singular respecto de los Hogares Pobres y No Pobres. En tanto miembros de los hogares en peores condiciones según la medida de N.B.I. todos aportan. El cuadro dentro del subgrupo por sexo y jefatura según nivel, para observar miembros con bajo o sin aporte.

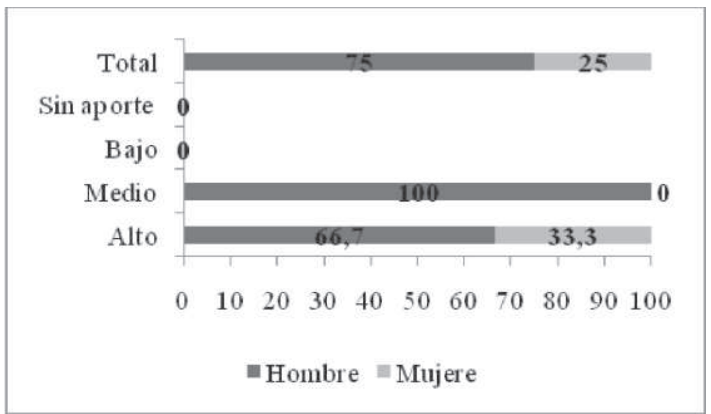

Figura 6. Aportes por sexo jefes de hogar en pobreza extrema (\%) 
Como en los dos casos anteriores, el aporte masculino se incrementa al pasar de alto a medio. En el caso de la mujer mayor no es que descienda su aporte, en tanto en este grupo la cabeza de hogar en su totalidad hace su aporte como alto.

En la totalidad del grupo de hogares en Pobreza extrema (Jefaturas de personas mayores y de no mayores) se observa ahora el peso contundente de dependencia que tienen estos hogares respecto de sus cabezas de hogar, en un $80 \%$ de personas mayores.

Tabla 6. Aportes Jefes Grupo pobreza extrema (\%)

\begin{tabular}{cccccccc}
\hline Sexo & Alto & Medio & Bajo & Subtotal & $\begin{array}{c}\text { Sin } \\
\text { aporte }\end{array}$ & $\begin{array}{c}\text { Sin } \\
\text { información }\end{array}$ & Total \\
\hline Hombre & 40 & 20 & 0 & 60 & 0 & & \\
Mujer & 20 & 0 & 0 & 20 & 0 & & \\
Total & 60 & 20 & 0 & 80 & 0 & 20 & 100 \\
\hline
\end{tabular}

Mientras, por N.B.I. no se encuentra dependencia económica de los mayores respecto de otros miembros del hogar, donde el arreglo familiar más frecuente, luego del aporte alto, es de equilibrio entre aportes, es decir el mayor aporta $50 \%$ o cerca de $50 \%$, y los otros miembros el $50 \%$ como caso minoritario: mientras el $75 \%$ de cabezas de hogar (masculinas y femeninas) aportan la totalidad de sus ingresos, el $25 \%$ restante lo hace con aporte medio.

La incidencia de estos hogares con jefatura mayor sobre la totalidad del grupo en pobreza extrema alcanza un $80 \%$ en este estudio. Si por el índice de N.B.I. la pobreza y la pobreza extrema sumadas dan un $25,61 \%$, parecería que se está en un mundo mejor, de minorías "desaventajadas" como suelen decir, y en contradicción con estudios de pobreza, donde son ejemplares los de Alma Mater, y el de PNUD, ya citados.

El matiz también introducido sobre las variables e indicadores para la región ya permiten minimizar el peso de algunos de estos indicadores, que siendo en general significativos, de modo relativo, como en nuestro caso, varían en su ponderación: habitabilidad de la vivienda y el lugar, como de servicios públicos domiciliarios jalonan hacia abajo las N.B.I. y esto ya saca de los horizontes entre conjuntos superiores y subordinados y excluidos, amplios conjuntos de población: unos ascienden con vértigo en esta escala al contar en sus viviendas y barrios con universalidad de servicios domiciliarios, pisos y paredes en "material", aunque la cifra de sus expectativas, acabalgada sobre sus ingresos ordinarios, corresponda al día día.

Aquí no se capturan los hogares de ciclo inicial, con pocos hijos y padres jóvenes y escolaridad entre estándar y algo más baja. Y viceversa ocurre: hogares con medianos y altos ingresos caen en pobreza por clima educativo del hogar, pero ya se ha mostrado con que variables y que hogares están en que N.B.I.
Sin embargo, las N.B.I. permiten generar, aún con estas limitaciones básicas, unas correspondencias coherentes con los noveles verticales entre No Pobres, Pobres y en Miseria:

Para el conjunto de los grupos (con jefes mayores y no mayores hombres y mujeres), el tamaño de los hogares presenta la siguiente composición:

Tabla 7. Promedio personas por hogar según grupo

\begin{tabular}{cc}
\hline Hogares & Promedio \\
\hline No pobres & 3.47 \\
Pobres & 5.13 \\
Pobreza extrema & 6.60 \\
\hline
\end{tabular}

Estas proporciones están sujetas a las composiciones familiares o de hogar de un universo concentrado en personas mayores.

Además, han sido claramente señaladas como incidentes para la no pobreza y la pobreza (incluida la pobreza extrema), entre otros, por Oscar Fresneda en una compilación de estudios y exposiciones del PNUD (2000).

Pero, obvio, no es el índice de natalidad sobre el que se montan los arreglos familiares por sí solos los que definen una situación estructural en la sociedad: no es la mayor o menor fecundidad, sino la estructura de oportunidades, o de otra manera, la disponibilidad de recursos, de su democratización o concentración, en el que la fertilidad es un factor, sujeto a decisiones y responsabilidades individuales y de pareja, bajo mediaciones culturales y condicionamientos sociales, pero con alta cuota subjetiva frente a las ya no tan individualizadas opciones frente a los recursos.

Tabla 8. Promedio personas hogar por jefes mayores y no mayores

\begin{tabular}{lcccccc}
\hline & \multicolumn{3}{c}{ Jefes mayores } & \multicolumn{4}{c}{ Jefes no mayores } \\
\hline Grupos & Total & M & F & Total & M & F \\
No & & & & & & \\
pobres & 3,41 & 3,27 & 3,64 & 3,56 & 3,12 & 4,25 \\
Pobres & 4,71 & 5 & 3,88 & 5,56 & 5,56 & 5,56 \\
Pobreza & 5,5 & 6 & 4 & 0 & 0 & 0 \\
extrema & & & & & & \\
\hline
\end{tabular}

Discriminados los grupos por la edad de las jefaturas y sexo, persiste la tendencia de menos integrantes a más al descender de no pobres a miseria; una diferencia entre 
jefaturas por edad, es la menor proporción en sus hogares que las de no mayores. Las jefaturas femeninas de no mayores son las que presentan los mayores promedios.

En los cuadros de pobreza extrema para jefes no mayores no se consignaron los datos por estar representados por un solo hogar de todo el conjunto en miseria, y este hogar tiene 11 miembros.

Se agrega que una tendencia similar en promedios por ingresos se presenta reagrupando por no pobres, pobres y en pobreza extrema discriminados por los filtros de un salario mínimo, 2 salarios mínimos.

En lo fundamental, se cumple para los tres primeros rangos de menores ingresos.

Aporte de personas mayores según nivel de ingresos del hogar

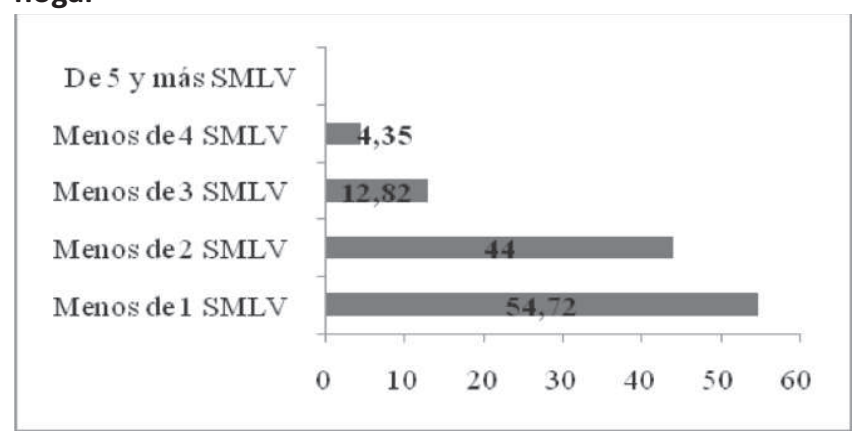

Figura 7. Alto aporte jefes hogar según salarios del hogar (\%)

La figura muestra una progresión interesante sobre el aporte de las personas mayores cabezas de hogar según niveles de ingreso del hogar por salarios: es de proporción inversa, como se ha señalado ya para los grupos por N.B.I de no pobres, pobres y en miseria, es decir, entre más se baja de no pobre a pobre incluida miseria, el aporte del adulto mayor es más significativa: es como obvio, al pensar que la sumatoria de los hogares por salarios entre más alta sea, más se diversifica entre los miembros aportantes, de tal manera que así el adulto mayor aporte la totalidad de su ingreso, este es apenas una fracción del total cuando se trata de los mayores ingresos por hogar.

Sin embargo, aquí hay que tomar en la cuenta, que al situarnos en los ingresos, están todos los grupos en cada rango o de salarios mínimos legales vigentes (para en este caso, el año de 2005). En ingresos de hasta un salario mínimo o algo menores están no pobres, pobres y pobreza extrema, e igual para todos los rangos.

Pero sean del grupo que sean según las N.B.I., los hogares de menor ingreso dependen más de los adultos mayores, hasta desaparecer o invisibilizarse en el conjunto, cuando los hogares por ingresos totales de sus miembros superan la franja de más de cinco salarios mínimos.

Invisibilizarse no significa inexistencia: grandes fortunas en Armenia son de viejos patriarcas, pero no todos los patriarcas y matriarcas tienen fortunas, salvo excepciones en un régimen que confirmado por muchas agencias, hace expresar de entrada al profesor colombiano Jaime Tenjo, apoyado en estudios del Banco Mundial, que:

"El tema de la equidad es de fundamental importancia en el proceso de desarrollo de los países y en las políticas para promoverlo. No solo es importante que los países crezcan, sino también que los beneficios del crecimiento económico lleguen a todos los estamentos de la población, especialmente a los más pobres.

Colombia tiene la poco honrosa distinción de tener uno de los mayores grados de desigualdad de los ingresos en el mundo (2)" (Tenjo y Galarza, 2004, 1).

Tabla 9. Aportes jefes hogar según ingresos (\%)

\begin{tabular}{cccc}
\hline Salarios & Alto & Medio & Bajo \\
\hline Menos de 1 SMLV & 54,72 & 34,9 & 3,77 \\
Hasta 2 SMLV & 44 & 22,4 & 0 \\
Hasta 3 SMLV & 12,82 & 35,9 & 0 \\
Hasta 4 SMLV & 4,35 & 52,2 & 8,7 \\
De 5 y mas SMLV & 0 & 60 & 20 \\
\hline
\end{tabular}

La tabla de referencia según salarios, que agrupa de acuerdo a los montos, por el nivel de aporte, está construido con los porcentajes de las jefaturas de hogar de adultos mayores (hombres y mujeres), que en cada rango perciben estos ingresos, así, de los jefes mayores que alcanzan hasta un salario mínimo o algo menos, el 54,72\% aportan alto; el $35 \%$ medio, y el 3,77 \% bajo; e igual para las otras categorías de ingresos.

Como se ha expresado, en alto aporte, en la medida en que es mayor el ingreso del hogar, disminuye el aporte alto de los cabezas de hogar mayores, pero tiende a aumentar el aporte medio de manera significativa.

La dependencia, como por las N.B.I., en este caso aumentaría o aumenta con mayores ingresos del hogar para los adultos mayores, aunque de manera importante, aporten hacia arriba con nivel medio, lo que configura unos arreglos familiares de corresponsabilidades económicas directas entre los miembros mayores y los menores de 60 años: 
Tabla 10. Suma aportes alto y medio jefes hogar según ingresos de hogar

\begin{tabular}{cccc}
\hline Salarios & Alto & Medio & Total \\
\hline Menos de 1 SMLV & 54,72 & 34,9 & 89,63 \\
Hasta 2 SMLV & 44 & 22,4 & 66,4 \\
Hasta 3 SMLV & 12,82 & 35,9 & 48,72 \\
Hasta 4 SMLV & 4,35 & 52,2 & 56,52 \\
De 5 y mas SMLV & 0 & 60 & 60 \\
\hline
\end{tabular}

Para todos los rangos, los aportes son claves, y a pesar de la dependencia económica, ella sola en blanco y negro vista, encubre matices sustantivos para el desarrollo de los miembros de la familia: El mantenimiento de la base familiar, el cuidado de los nietos, la gestión administrativa del hogar, permiten liberar y desahogar de funciones y compromisos a los familiares de generaciones menores para su vinculación al estudio y al trabajo (lo contrario también ocurre: más que liberarlos, por las condiciones estructurales de acceso a recursos, de vincularse de manera preferente a la consecución de recursos económicos, y no a invertir en capital humano, o en términos de Sen, a cultivar y apropiar capacidades).

Además, es difícil ponderar entre varios factores simultáneos como el de la independencia económica y el estatus que otorga el ser aportante económico directo, y las posibilidades de arreglos familiares que permiten que los adultos mayores (un segmento de ellos) puedan liberarse de las cargas económicas, por un relevo ético y social, aunque de índole familiar, por generaciones menores que retribuyen, para no mencionar los ciclos de vida activa y salida de ella para abrir campo a las generaciones menores, que de otra manera no sobrevirían.

En lectura gruesa, el cuadro anterior de aportes según ingresos sugiere varios nodos:

- Que aún para los hogares de ingresos más altos, la alta dependencia de los aportes de adultos mayores, implica grados de precariedad para afrontar las responsabilidades vitales, en un mundo de mercado, ante la ausencia de estos mayores.

- Elemental, pero siempre significativa en los estudios, que esta precariedad aumenta entre menores los ingresos sobre todo en estos hogares multigeneracionales dependientes del adulto mayor.

- Aunque no se ha consignado, y a pesar de corresponder a un número reducido de hogares, su proporción si es considerable: un aproximado $34 \%$ de hogares reciben apoyo de hijos fuera del hogar: de estos, el $81 \%$ que están en Colombia, y sus aportes son equivalentes a la estructura de ingresos y oportunidades nacionales, es decir, menores que los aportes que hacen los del exterior, que representan un 19\%; y que en conjunto apoyan o tributan de manera preferencial para el núcleo de hogares que declaran y se infiere, no reciben salarios, o los reciben en montos casi invisibles, en aproximadamente un $15 \%$ a $16 \%$, fuera de aquellos que ignoran de montos salariales al responder en este estudio, pero saben de aportes específicos de sus miembros para manutención, servicios, etc.

\section{Consumos}

Para los consumos, se ha realizado la lectura de los hogares desde los grupos y categorías más generales a las más específicas. Los cuadros presentan una dinámica concebida desde el momento de la encuesta por dos años hacia atrás, situando por lo menos en este rango el devenir económico de los hogares.

Con 7 rubros y 4 posibilidades que los cruzan, las lecturas pueden ser múltiples. Para los tres primeros rubros en los jefes no mayores se ha presentado una disminución notable, mientras hasta el cuarto rubro para los hogares con cabezas mayores, siendo más drástica la caída en el consumo de alimentos básicos en los de no mayores que en mayores; para los mayores, los gastos en salud han debido incrementarse, mientras disminuye hasta no consumo (gasto) en esparcimiento/diversión casi 12 puntos más en no consumo sobre los hogares más jóvenes.

Para el primer rubro es sistemático en el descenso en el acceso a los alimentos básicos, y para los hogares situados en miseria, salvo en salud, caen radicalmente o no se consumen; entre los no pobres y los pobres, los de otros alimentos, vestuario y calzado, y transporte son de menor impacto en los pobres, no así en salud. En los de pobreza extrema la salud simultáneamente ha debido aumentar en gasto.

Recordemos que la salud, para el régimen contributivo los copagos y cuotas moderadores encarecen el servicio y dificultan el acceso regular a ella.

Para esta categoría, en los alimentos básicos ya es notable la progresión negativa; con una menor caída la de otros alimentos de los pobres frente a los no pobres, y que por el contrario aumenta de manera leve, como forma compensatoria. En vestuario y calzado en los pobres es menor la caída que en los pobres, pero que se reflejó cuando aumenta de modo ligero, aún por debajo de los no pobres.

En los no pobres la baja más significativa en este rubro puede estar expresando un mayor impacto de un consumo entre regular y amplio, que ha debido encogerse, mientras para los pobres, una "disciplina" de moderación en su gasto, o de forzosa limitación histórica, hace que su economía, aunque se resienta también en este rubro, tenga efectos menos 
devastadores que por ejemplo en sus alimentos.

En salud para los grupos de esta categoría de no pobres y pobres, aproximadamente para cada $50 \%$ se mantiene su consumo similar; para la otra mitad en los pobres y no pobres , una proporción considerable ha debido incrementar el gasto en salud (29\%), pero para los pobres el restante grupo de hogares ha debido disminuir su acceso.

En educación para los pobres, el mayor porcentaje se establece entra mantenerse e incrementarse su consumo (o inversión); mientras para los no pobres, siendo menor en la parte de caída en acceso, aumenta de forma notoria su no consumo, que puede estar relacionado a cumplimiento de ciclos básicos.

El número de integrantes aumenta multigeneracionalmente en los hogares no pobres y en los hogares en pobreza extrema, con aumento de generaciones menores y por lo tanto, de mayores demandas al sistema escolar.

Se ha dejado el cuadro de miseria, pero con la salvedad ya hecha que está referido a un solo hogar, con todas las precauciones que para su lectura esto entraña.

Se debe tener en cuenta, en la comparación de las categorías, el menor nivel de respuesta en los hogares no pobres, de los que respondieron el $86 \%$.

Para los tres primeros rubros, la caída se revela mayor para los hogares no pobres que para los pobres, mientras que para los hogares en pobreza extrema el descenso es abrupto y muy grave. Pero observando la fila de no variación, se presenta una mayor estabilidad en os hogares no pobres que en los pobres, donde estos últimos han debido aumentar los consumos en alimentos y vestuario y calzado por encima de los no pobres casi en el mismo monto en que han caído para estos; una posible razón puede radicar en el incremento de miembros en los pobres, con la demanda consiguiente de estos nuevos miembros.

En transporte salud y educación cambia la tendencia al mostrarse un decremento en los pobres, y por el contrario ha implicado incremento en los hogares más afectados por la desigualdad, los en pobreza extrema donde las demandas y consumos en salud y educación son superiores, por el incremento mucho mayor de nuevas generaciones.

Comparando los dos grupos de jefes no mayores y jefes mayores en sus categorías, se tiene:

$\S \quad$ Entre los no pobres, en los tres rimeros rubros se comportan de forma muy similar, aunque en los hogares presididos por viejo se da un mayor incremento en la demanda de otros alimentos distintos a los básicos.

$\S \quad$ En transporte y en salud es mayor ahora el descenso en los hogares no pobres de las cabezas de hogar mayores.

$\S \quad$ Entre los pobres no mayores y los pobres con mayores, para los primeros es muy significativa la caída en el consumo de los alimentos básicos, que puede estar significando el peso de los aportes de los viejos, en este caso ausentes como cabezas de hogar, lo que los convierte en mucho más frágiles.

En los otros rubros, lo pobres de no mayores jefes presentan una mayor estabilidad y un aumento en salud y educación: estos hogares de jefaturas no mayores tienen, de acuerdo al análisis inicial de promedios de integrantes, uno mucho más alto que el de los hogares con jefatura de adultos mayores.

Cuando el cruce entre consumos se efectúa con ingresos, a modo de ejemplo, se encuentra que, para el primer rubro de alimentos básicos:

Tabla 11. Consumo alimentos básicos según ingresos del hogar

\begin{tabular}{lc}
\hline $\begin{array}{c}\text { SALARIOS DEL } \\
\text { HOGAR }\end{array}$ & $\begin{array}{c}\text { DISMINUCIÓN ALIMENTOS } \\
\text { BÁSICOS }\end{array}$ \\
\hline DE O A INFERIOR A 1 & 72,06 \\
1 SMLV & 70,75 \\
2 SMLV & 57,6 \\
3 SMLV & 58,3 \\
4 SMLV & 52,17 \\
5 SMLV & 28,57 \\
6 Y MÁs & 27,2 \\
\hline
\end{tabular}

Esta lectura, de la que se ha tomado un rubro, tiene un comportamiento aproximadamente similar para otros rubros.

Consumos a través de ingresos resulta mucho más sensible para entender el comportamiento económico de los hogares, desde el que es dable observar, independiente de las tres categorías de No Pobre, Pobre y en Pobreza Extrema, ya analizadas, y que han arrojado resultados consistentes, pero en este caso específico no tan precisos como ahora.

\section{CONCLUSIONES}

El total de personas que habitan el total de hogares es 1.439, de los cuales el $36.1 \%$ corresponde a la población mayor de 60 años de edad. El promedio de personas por hogar es de 3.7.

Diferente a los hogares de constitución reciente, el ciclo de vida de los hogares en Armenia, en una alta proporción son presididos por adultos mayores, lo que hace que la pirámide de edad sea diferenciada, a pesar de la multigeneracionalidad, y también mejor, precisamente por ella, de las pirámide de base más ancha y cúspide más estrecha.

Lo que representa que casi el $50 \%$ de los hogares reciban aportes, que el $31 \%$ de todos los hogares basen su reproducción económica de los adultos mayores; y que una sexta parte $(15 \%)$, dependa en un $50 \%$ de los ingresos totales de sus hogares, del aporte del adulto mayor. 
Importante señalar que, en correspondencia con trabajos de la CEPAL, el aporte de los adultos mayores para el sostenimiento y reproducción económica de los hogares es considerable, desde un casi $50 \%$ en adelante, y de modo absoluto para los hogares situados en las franjas de pobreza y miseria.

En general y para los hogares, sobre todo los de no pobres, los niveles de dependencia económica de las mujeres mayores es más alta.

De acuerdo a los ingresos del hogar, medidos por salarios mínimos, los jefes mayores que alcanzan hasta un salario mínimo o algo menos, el 54,72\% aportan alto; el $35 \%$ medio, y el 3,77\% bajo; e igual para las otras categorías de ingresos.

El aporte, en la medida en que es mayor el ingreso del hogar, disminuye el aporte alto de los cabezas de hogar mayores, pero tiende a aumentar el aporte medio de manera significativa.

La dependencia, como en el caso de las N.B.I. aumenta, pero ahora por los mayores ingresos del hogar, para los adultos mayores, aunque de manera importante, continúen aportando con nivel medio, lo que configura unos arreglos familiares de corresponsabilidades económicas directas entre los miembros mayores y los menores de 60 años.
Establecidos los ingresos, de manera independiente a la categoría de no pobre, pobre o en miseria, entre más bajo es el ingreso del hogar, mucho mayor es el aporte del adulto mayor, en consecuencia, generando un círculo de fragilidad por la alta dependencia del hogar sobre sus adultos mayores.

El mayor número de miembros -ciclo vital de los hogaresaumenta al descender en la escala de no pobres a pobres y miseria, donde los hogares de jefaturas de no mayores, presentan mayores impactos negativos en sus consumos, que en las presididas por adultos mayores.

Por sexo, la tendencia es la de mayor fragilidad de los hogares con jefatura femenina, y para los dos grupos de hogares de mayores y no mayores en sus cabezas de hogar.

Una proporción importante fortalece en unos casos, o depende enteramente de los aportes que miembros de la familia (hijos) externos al núcleo, sea residentes en otros lugares de Colombia, o en el exterior, hacen a sus hogares de origen, configurando una modalidad de compromisos y arreglos familiares al parecer cada vez más extendida, como recurso actual ante las penurias y limitaciones de los hogares. Estos recursos en las cantidades de aporte están claramente diferenciados para los hijos externos en Colombia, y los que están en el exterior: los segundos son mucho más cuantiosos.

\section{BIBLIOGRAFÍA}

Aranibar, P. (2001). Acercamiento conceptual a la situación del Adulto Mayor en América Latina. Santiago de chile, En: Serie Población y desarrollo, CELADE-FNUAP.

Comisión Económica para América Latina y el Caribe. (2003) América latina y el Caribe: el envejecimiento de la población 1950-2050. Boletín demográfico.

DANE. (2003) Carta Estadística 2002. Departamento Administrativo de Planeación. Gobernación del Quindío.

Del Popolo, F. (2001). Características sociodemográficas y socioeconómicas de las personas de edad en América Latina. Santiago de chile, En: Serie población y desarrollo, CELADE.

Fresneda, O. y otros. (1991) Pobreza, violencia y desigualdad: retos para la nueva Colombia. Bogotá: Programa de las naciones Unidas para el Desarrollo.

Girado, N., Fernández, A y De la Rosa, J. (2008). “Dependencia económica y social de los adultos mayores de 60 años en la ciudad de Armenia, Quindío". En: Revista de investigaciones Universidad del Quindío, (18), 108-121.

Ministerio de Salud. Dirección General de Promoción y Prevención. Programa de prevención de enfermedades crónicas y degenerativas. Primera edición. Bogotá, 1997.

Ministerio de Salud. Resolución Número 03997. Santa fe de Bogotá, D.C. Octubre de 1996.

Ministerio de Salud. Resolución Número 412. Santa fe de Bogotá, D.C. Febrero de 2000.

Núñez, J. y Espinosa, S.(2005, Noviembre) Pobreza y Protección social en Colombia. PNUD - Asdi. Bogotá,

Pérez, J. (2000 Octubre, 25 Miércoles) "Envejecimiento poblacional y dependencia. Una perspectiva desde la necesidad de cuidados". En: II Jornada Gerontológica de Recursos Asistenciales. Pamplona.

Pizarro, R. (1999). Vulnerabilidad Social en América Latina y el Caribe, Santiago de Chile. CELADE, LC/DEM/R. 29,

Programa de las Naciones Unidas para el Desarrollo (2004). Eje cafetero. Un pacto por la región". Informe Regional de Desarrollo Humano. Manizales: Litocamargo.

Sen. A. (2000). Desarrollo y libertad. Bogotá, Planeta. 